\title{
Advanced Timed Influence Nets with Random Time Delay for Weapon System-of-systems Combat Networks
}

\author{
ChunXiao $\mathrm{Fu}^{1, \text { a }}$, Jiang Jiang ${ }^{1, \mathrm{~b}}$, KeWei Yang ${ }^{1, \mathrm{c}}$ \\ ${ }^{1}$ National University of Defense Technology College of Information System and Management \\ ChangSha, China \\ achunxiaosky@hotmail.com, ${ }^{\mathrm{b}}$ jiangjiangnudt@163.com, ${ }^{\mathrm{c}}$ kayyang27@gmail.com
}

Keywords: timed influence net; random time delay; weapon system-of-systems combat networks

\begin{abstract}
To meet the needs of the joint operation under the uncertain information conditions, the description and effectiveness of weapon system-of-systems combat networks has been widely researched. The Timed influence nets(TIN) is an useful tool to describe the uncertain causal relations in the weapon system-of-systems combat networks and has been used for the effectiveness evaluation in alternative military actions. However the uncertain time delay effect is not well considered and computed in the TIN. In this paper, the Random Timed Influence Nets(RTIN) is proposed to introduce the random time delay in the TIN. First, it is introduced that the basic concepts and algorithms used in the RTIN model including the time delay parameter, the belief sequence and the belief-spread algorithm. And then an instance is given in this paper to demonstrate the RTIN modeling process and the result of effectiveness evaluation using the belief spread algorithm.
\end{abstract}

\section{Introduction}

In the joint operations, it is really important to reach the desired effect of the combat target selection to help the commanders to make decisions. A good way is to model and analyze the uncertain causal relationship between operations and effects. And Bayesian Networks (BN) is frequently used in that issue. However the conditional probability table in the BN is always hard to get. Rosen J A[1] introduced the idea of CAST (causal strength), and came up with the influence nets (IN) modeling method. As the nodes increase, the parameters we need in IN are not exponentially increased. Both $\mathrm{BN}$ and IN describe static relationships between parameters, some researchers tried to introduce the time factor and uncertain factor into IN. [2] Wagenhals and his team [3] introduced the time structure in IN modeling, which is called timed influence nets.[4] TIN has been widely used for Effects-based Operations that mainly for the evaluation of operations.

There are also disadvantages in the TIN modeling method. And the Dynamic influence nets modeling and some other methods are introduced to make some improvement for influence nets. [5]However, in the military combating process, there are random time delays between the operations and effects, which is unable to describe in TIN. Thus in this paper, we introduced the random time delay and random belief sequence parameters into TIN, which is called random timed influence nets (RTIN). The mathematic description for RTIN and the belief spreading algorithm is elaborated. And an example is given to demonstrate the modeling process and the result of RTIN.

\section{Relative Study}

Bayesian Network is a probability networks model came up by R.Howard and J. Matheson. In Bayesian Networks, the uncertain factors are described as the probabilities which can be deduced and calculated. While modeling for realistic situations, there are also some disadvantages in Bayesian Networks, such as the limitation for calculation time and the amount of given information.

To improve the usability of Bayesian networks, Chang introduced CAST parameters and came up Influence Nets. In Influence Nets the causal relationship between nodes. While calculating, the CAST parameters can be converted to the Conditional Probability Table, which can be used in Bayesian Networks probabilities deduce. 
Influence Nets is an improved static influence diagram, which cannot reflect the real dynamic relationship between nodes. Thus, Wagenhals introduced the concept of time parameter and came up the modeling method of Timed Influence Nets through setting time parameters in the static influence nets.

A typical Timed Influence Nets is shown in Fig 1:

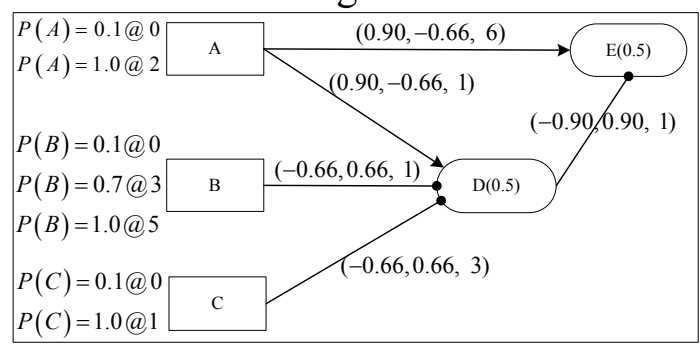

Fig 1 Timed Influence Nets

Timed Influence Nets is also described as a Directed Acyclic Graph(DAG) for topological structure, in which the nodes reflect the random variable and the directed edges between the nodes reflect the causal relationship.

A Timed Influence Networks can be described by the following six elements

$$
I N=<V, E, C, B, D, A>
$$

$\mathrm{V}$ : The set of the nodes in TIN.

E: The set of the edges in TIN.

$\mathrm{C}$ : The set of CAST parameters between nodes. For $\forall \mathrm{e} \in \mathrm{E}, \exists \mathrm{c} \in \mathrm{C}$, satisfies $f_{1}: e \mapsto c$, in which, $\mathrm{C}=\{(\mathrm{h}, \mathrm{g}) \mid-1 \leq \mathrm{h}, \mathrm{g} \leq 1\},(\mathrm{h}, \mathrm{g})$ is the expression of CAST.

B: The set of baseline probabilities or prior probabilities.

D: The set of time delay on child nodes produced by parent nodes in TIN.

A: The input of the TIN, which means the set for the belief sequence of the root nodes.

There are some disadvantages of Timed Influence Nets. In real situations, the time delays are always not certain values but random values which also lead to the uncertainty of belief values.

In this paper, based on the random factors of time delay and the uncertainty of belief value, an improved Timed Influence Nets is introduced to take the random factors of time into consideration.

\section{Description for RTIN}

The disadvantages of Timed Influence Nets can be seen from the preceding part. Two new concepts are introduced in this paper, Random Time Delay and Random Belief Sequence.

An example of the Random Timed Influence Nets is shown in Figure 2.

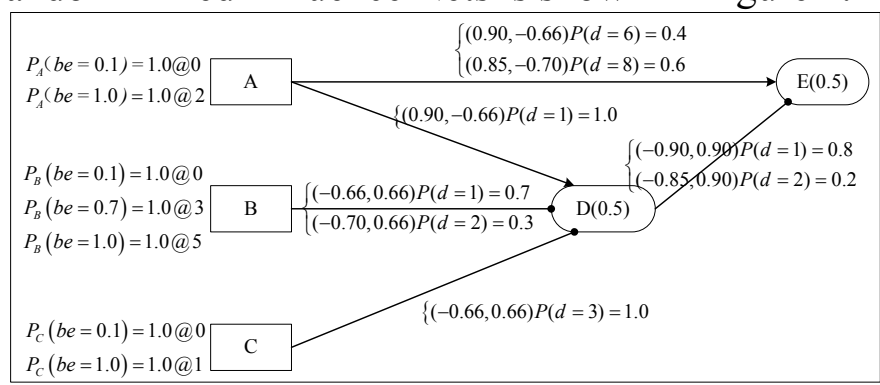

Figure 2 Random Timed Influence Nets

Definition 1. Random Time Delay. In Random Timed Influence Nets, the time delay corresponded to any edges is a random variable.

Definition 2. Random Belief Sequence. In Random Timed Influence Nets, the taking value process of any node is a random sequence.

Here the random belief sequence is the random sequence be $(\mathrm{t}) \sim P_{t}\left(W_{t}\right)$ defined in time interval $[0, \mathrm{Mt}]$. In that, be(t) means the random variable corresponded to the node's belief at the time of $\mathrm{t}$. Finite set $W_{t}$ is the range of be $(\mathrm{t})$. Any element in $W_{t}$ means the value that the belief of the nodes can be. $P_{t}\left(W_{t}\right)$ is a discrete probability distribution defined on set $W_{t}$. During the time [0, 
$\mathrm{Mt}$ ], the time interval that the belief updates is denoted as TI. And the time that belief updates is denoted as TP. The TP is only derived from TL. The max time of TP is denoted as $t_{\max },\left[0, t_{\max }\right]$ is the standard time valuing interval.

If the $T I=\left\{\left[t_{1,1}, t_{1,2}\right], \ldots\left[t_{n, 1}, t_{n, 2}\right]\right\}$, then $T P=\left\{t_{1,1}, t_{2,1}, \ldots, t_{n, 1}\right\}\left(t_{i, 1}<t_{i, 2}, t_{i+1,1}-t_{i, 2}=\right.$ $1, i=1,2, \ldots, n)$.

After introducing the concepts, the Influence Nets is described as follow:

$$
I N=<V, E, C, B, D, A>
$$

$\mathrm{V}$ : The set of the nodes in TIN. Each node corresponds to a Boolean random variable: $\forall \mathrm{v} \in$ $\mathrm{V}, X_{v}=0,1$; nodes are composed of root nodes $V_{r}$ and non-root nodes $V_{m}$. And the probabilities of variable $\mathrm{X} P(\mathrm{X}=1), \mathrm{P}(\mathrm{X}=0)$ are denoted as $\mathrm{P}(X)$ and $P(\neg X)$ respectively.

$E$ : The set of the edges in TIN.

$\mathrm{C}$ : The set of CAST parameters between nodes. For $\forall \mathrm{e} \in \mathrm{E}, \exists \mathrm{c} \in \mathrm{C}$, satisfies $f_{1}: e \mapsto c$, in which, $\mathrm{C}=\{(\mathrm{h}, \mathrm{g}) \mid-1 \leq \mathrm{h}, \mathrm{g} \leq 1\},(\mathrm{h}, \mathrm{g})$ is the expression of CAST.

$\mathrm{B}$ : The set of baseline probabilities or prior probabilities, in which the root nodes correspond to the baseline probabilities and the non-root nodes corresponds to the prior probabilities, and they mean without the interference of other nodes, the probabilities of the nodes. $\forall \mathrm{v} \in \mathrm{V}, \exists \mathrm{b} \in \mathrm{B}$ Satisfies $f_{2}: v \mapsto b, 0 \leq b \leq 1$;

D: The set of time delay on child nodes produced by parent nodes in RTIN, $\forall \mathrm{e} \in \mathrm{E}, \exists \mathrm{d} \in \mathrm{D}$ Satisfies $f_{3}: e \mapsto d, \mathrm{~d} \sim \mathrm{P} d_{e}\left(\Omega_{e}\right), \Omega_{e}$ is nonnegative integer set, which means the range of $\mathrm{d}$, and .

$\mathrm{P} d_{e}\left(\Omega_{e}\right)$ is the discrete probability distribution defined on set $\Omega_{e}$.

The time-delays corresponded to edges are independent from each other.

A: The input of the Random Timed Influence Nets which means the set of all root nodes' beliefs.

For $\forall \mathrm{v} \in V_{r}, \exists \mathrm{a} \in$, satisfies $f_{6}: v \mapsto a, a=\left\{\left\langle\left[t_{i, 1}, t_{i, 2}\right], P_{v i}\left(W_{v i}\right)\right\rangle, 0 \leq b e_{v i} \leq 1, t_{i, j} \in N, i=\right.$ $0,1,2, \ldots, n, j=1,2\}$, in which, $\left\{\left[t_{i, 1}, t_{i, 2}\right]\right.$ denotes 1 unit's time interval, $t_{i, 1}=t_{i, 2}-1 .<$ $\left[t_{i, 1}, t_{i, 2}\right], P_{v i}\left(W_{v i}\right)>$ denotes: at any time during $\left[t_{i, 1}, t_{i, 2}\right]$, the belief of node $\mathrm{v}$ is the probability of $X_{v}=1$ at the time of $t_{i, 1}$ which is $P_{v i}\left(W_{v i}\right) . P_{v i}\left(W_{v i}\right)$ is the discrete probability distribution defined on the set $W_{v i} ; W_{v i}$ is composed of the value that v's belief can be. And the belief value varies in different time intervals. Especially in the first time interval $\left[t_{0,1}, t_{0,2}\right]$, the belief of node $\mathrm{v}$ equals to the prior probability, $P_{v 0}\left(b e_{v o}(0)=f_{2}(v)\right)=1, W_{v 0}=\left\{f_{2}(v)\right\}$.

Through the definition of the input of the improved Timed Influence Nets, it is known that A is a set composed of random belief values of nodes. Here defines the

Belief Spreading Moment. as the time when the root node's belief value changes.

Belief Spreading Algorithm.The Belief Spreading Algorithm is designed to get the belief sequence of the non-root nodes. In this part, the main idea of the algorithm is firstly introduced, then the calculating method is given.

The algorithm is under the condition of the assumption that the son nodes and the parent nodes are independent from each other. Firstly the nets should be divided into some Basic Nets. Next through marking the nodes one by one to make sure all nodes in the nets are able to be calculated, then we can get the belief sequence of nodes.

The Basic Nets means the nets that only have one non-root node and no intermediate nodes in it. In figure 3 the example of Basic Nets is given.

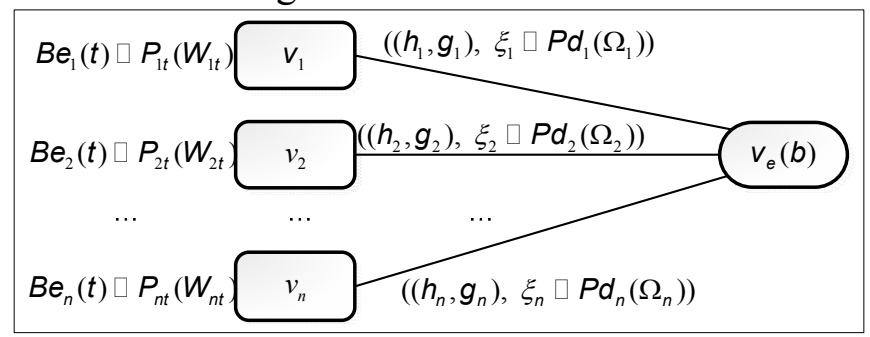

Figure 3: Example of Basic Nets

In the nets, $v_{e}$ corresponds to the random variable $\mathrm{Y}$, and its baseline probability is $b_{Y}$; root nodes $v_{i}$ correspond to random variables $X_{i}$ and their prior probabilities $p_{i}=f_{b}\left(v_{i}\right)$. The 
influence strength from $v_{i}$ on $v_{e}$ is $\left(h_{i}, g_{i}\right)$; the time delay $d_{i Y}: P d_{i}\left(\Omega_{i}\right)$, the set of time delay $\Omega_{i}=\left\{d_{i 1}, d_{i 2}, \ldots, d_{i k_{i}}\right\}$, the input of $\left.v_{i}:\left\{B e_{i}(t): P_{i t}\left(W_{i t}\right) \mid t \in\left[t_{1,1}^{i}, t_{1,2}^{i}\right] \cup, \ldots, \cup t_{n i, 1}^{i}, t_{n i, 2}^{i}\right]\right\}$, $W_{i t}=\left\{p_{i 1}^{t}, p_{i 2}^{t}, \ldots, p_{i k_{i}}^{t}\right\}$ means at time of $\mathrm{t}$, the valuing set of $v_{i}$.

The known nodes refer to the nodes whose belief sequences are known. In the probabilistic reasoning process, the known nodes are marked. If all the parent nodes are marked, then the son node is resolvable. If all the root nodes in a basic net are marked, then the basic net is resolvable.

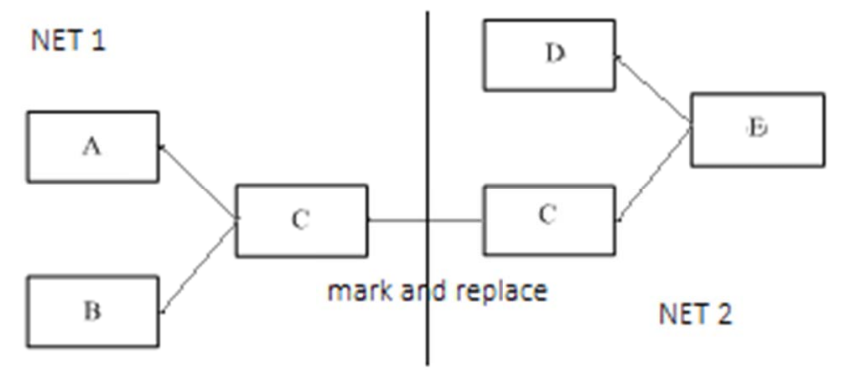

Figure 4: Idea diagram

The main idea diagram for getting the belief sequence of the nodes is showed in figure 4 . Firstly the whole net is divided into two basic nets, Net_1 and Net_2. In Net_1, all the root nodes are marked, so Net_1 is resolvable. In Net_2, node D is not marked and Net $\overline{2}$ is not resolvable. Firstly, get the belief sequence of $\mathrm{D}$, after calculating and mark $\mathrm{D}$. Then replace $\mathrm{D}$ in Net_ 2 with the marked node D in Net_1. And Net_2 becomes resolvable. Then the node E is resolvable and the belief sequence of $\mathrm{E}$ can be calculated.

\section{Calculating Method for the Belief Sequence of Leaf Nodes}

The root nodes decide the belief of the leaf node. And the uncertainty of the belief is decided by the uncertainty of the root nodes' belief combination. The uncertainty of the root nodes' belief combination is decided by the uncertainty of root nodes' belief spreading time and the uncertainty of the root nodes' belief values at spreading time. The uncertainty of the root nodes' belief spreading time is decided by the uncertainty of time delay.

At first calculate the leaf nodes' belief update time sequence $T P_{Y}=\left\{\tau_{0}, \tau_{1}, \ldots, \tau_{m}\right\}$, and select element $\mathrm{r}$ by sequence. And the related root nodes' belief spreading time vector $\mathrm{T}$ follows the probability distribution $T \sim P_{\tau}(\psi)$ and $\psi=T P_{1} \times T P_{2} \times \ldots \times T P_{n}$. Select elements from $\psi$ by sequence $T_{0}=\left[t_{1}, t_{2}, \ldots, t_{n}\right], P_{r}\left(T=T_{0}\right)=p_{r}$. Then get the random vector $\mathrm{W}$ from root node $R_{i}$ 's belief values at time $t_{i}$ in turn. W follows the probability distribution $W \sim P_{W \mid T}\left(W_{T}\right)$ and $W_{t}=$ $W_{i t 1} \times W_{i t 2} \times \ldots \times W_{i t n}$. After that select elements $W_{0}=\left[w_{1}, W_{2}, \ldots, W_{n}\right]$ and $P_{W \mid T=T_{0}}(W=$ $\left.W_{0}\right)=p_{W T}$. And the probability that at time $\tau$ that the leaf node's belief equals to $f\left(W_{0}\right)$ is $p_{T} p_{W T}$. Then traverse the set $\psi$ and get the belief distribution of leaf node at the time of $\tau$.

$$
f\left(W_{0}\right)=\sum x_{1} \sum X_{2} \cdots \sum X_{n} p_{1}\left(X_{1}\right) \times p_{1}\left(X_{2}\right) \times \cdots \times p_{1}\left(X_{n}\right) \times p\left(Y \mid X_{1}, \cdots, X_{n}\right)
$$

$X_{i}$ is the random variable corresponding to root node $R_{i} \cdot P\left(Y \mid X_{1}, \ldots, X_{n}\right)$ denotes the conditional possibility distribution, the method to calculate $P\left(Y \mid X_{1}, \ldots, X_{n}\right)$ is elaborated in the reference paper[1]

\section{Calculation Method for the Belief Spread Time's Probability Distribution of Leaf Nodes}

The belief spreading time of the root nodes is only related with the time delay. According to the independent relationship between the time delays between different nodes, the belief spreading times of the root nodes are independent from each other. Thus, at the moment of $\tau$, the random belief spreading time vector $\mathrm{T}$ 's probability distribution of root nodes could be defined as follow:

$$
P_{\tau}(T)=\prod_{i=1}^{n} P_{\tau_{i}}\left(T_{i}\right)
$$

In that, $T_{i}$ is the belief spreading time of root node $R_{i}, T_{i}$ follows the probability distribution on the belief update time sequence $T P_{i}: T_{i} \sim \operatorname{Pr}_{i}\left(T P_{i}\right), T P_{i}=\left\{t_{i 0}, \ldots, t_{i n_{i}}\right\}$. 
And suppose at the time of $\tau$, for root node $R_{i}$, the time delay is $d_{i}$ and the belief update time is $t_{i}$, then $\operatorname{Pr}_{i}\left(T_{i}=t_{i}\right)=P d_{i}\left(d_{i Y}=d_{i}\right)$. Traverse the random time delay set $\Omega_{i}$ we can get the probability distribution $T_{i} \sim P r_{i}\left(T P_{i}\right)$ that the belief spreading time of $R_{i}$ follows at the time of $\tau$.

As for the belief spreading time $t_{i}$ :

If $\tau-d_{i} \leq 0$, then $t_{i}=0$, and there is no effect from root node $R_{i}$ to the leaf nodes.

Otherwise, the effect from the root node $R_{i}$ at the time of $\left(\tau-d_{i}\right)$ can reach on leaf nodes. If $\left[t_{k, 1}^{i}, t_{k, 2}^{i}\right]$ exists in the input $a_{i}$ of node $R_{i}$, and $t_{k, 1}^{i} \leq \tau-d_{i} \leq t_{k, 2}^{i}$, then $t_{i}=t_{i k}=t_{k, 1}^{i}$, if not then $t_{i}=t_{i n_{i}}=t_{n_{i}, 1}^{i}$.

In the probability distribution of the root node $R_{i}$, if $\operatorname{Pr}_{i}\left(T_{i}=t_{i 1}\right)=p_{\mathbf{1}}$ and $\operatorname{Pr}_{i}\left(T_{i}=t_{i 2}\right)=$ $p_{2}$ satisfy $t_{i 1}=t_{i 2}=t_{i}$, then we need to merge the two equations into one:

$$
\operatorname{Pr}_{i}\left(T_{i}=t_{i}\right)=p_{1}+p_{2}
$$

\section{Calculation Method for the Conditional Probability Distribution for the Belief Values of Root Nodes}

The belief value of the root nodes is only related to the belief spreading time, for a given belief spreading time sequence $T_{0}=\left\{t_{1}, t_{2}, \ldots, t_{n}\right\}$, the belief values' vector W's conditional probability distribution of root node can be defined as follow:

$$
P_{W \mid T=T_{0}}(W)=\prod_{i=1}^{n} P_{i t_{i}}\left(W_{i}\right)
$$

In which, $W_{i}$ is the $R_{i}$ 's belief value at the time of $t_{i}$, and $W_{i}$ follows the root node $R_{i}$ 's random belief sequence $b e_{i}(t) \sim P_{i t}\left(W_{i t}\right)$, and $W_{i} \sim P_{i t_{i}}\left(W_{i t_{i}}\right), W_{i t_{i}}$ is the belief value set of $R_{i}$ at the time of $t_{i}$.

\section{Demonstration}

In a military operation, we need to destroy an airport's combating capability. And we use this instance to demonstrate the RTIN modeling and calculating process and result.

Establish a random timed influence nets. Firstly analyze the tasks of different levels and confirm the relationships between different tasks also tasks and effect to establish the structure of the nets. And then set the parameters in the nets.

In the nets we built, $t_{1}, t_{2}, t_{3}$ are the moments that tasks $\mathrm{A}, \mathrm{B}, \mathrm{C}$ start. Take the task $\mathrm{B}$ for example: if $\mathrm{B}$ is not executed, the airport pavement could be unusable for the probability of 0.01 . If $B$ is executed, $B$ can effect the belief of $E$ (contribute to the destroy of pavement) at 0.75 . And the time delay between $\mathrm{B}$ and $\mathrm{E}$ could be 5 time units $(40 \%)$ or 6 time units $(60 \%)$.

And here we consider two plans to execute the tasks:

PLAN A: $t_{1}=1, t_{2}=1, t_{3}=1$.

PLAN B: $t_{1}=2, t_{2}=1, t_{3}=1$.

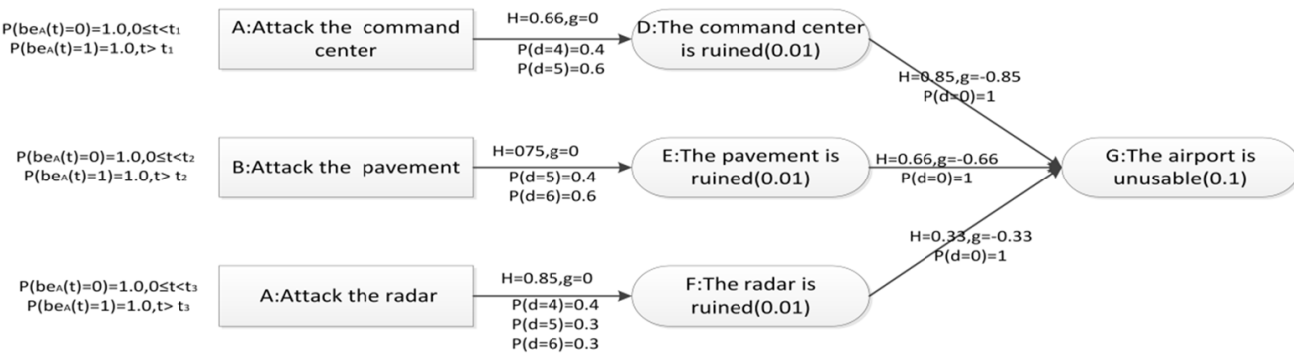

Figure 5 RTIN modeling combating capability of destroying airport

Calculation for the belief values of nodes.Firstly calculate the beliefs of $D, E$ and $F$, and then we can get the belief of G. From the belief-spreading algorithm, we can get the belief table of G: 
Table 1: Plan A

\begin{tabular}{|c|c|c|c|}
\hline $\begin{array}{c}\text { TIME } \\
\mathrm{t}\end{array}$ & Belief & Probability & $\begin{array}{c}\text { Average } \\
\text { belief } f_{\text {avg }}(t)\end{array}$ \\
\hline $0-4$ & 0.0078 & 1.00 & 0.0078 \\
\hline \multirow{4}{*}{5} & 0.0078 & 0.36 & \multirow{4}{*}{0.1462} \\
\hline & 0.2771 & 0.24 & \\
\hline & 0.0143 & 0.24 & \\
\hline & 0.4591 & 0.16 & \\
\hline \multirow{4}{*}{6} & 0.2271 & 0.18 & \multirow{4}{*}{0.4692} \\
\hline & 0.4591 & 0.42 & \\
\hline & 0.5405 & 0.12 & \\
\hline & 0.6095 & 0.28 & \\
\hline $7-10$ & 0.6095 & 1.00 & 0.6095 \\
\hline
\end{tabular}

Table 2: Plan B:

\begin{tabular}{|c|c|c|c|}
\hline TIME $\mathrm{t}$ & Belief & Probability & $\begin{array}{c}\text { Average } \\
\text { Belief } f_{\text {avg }}(t)\end{array}$ \\
\hline $0-4$ & 0.0078 & 1.00 & 0.0078 \\
\hline \multirow{2}{*}{5} & 0.0078 & 0.60 & \multirow{2}{*}{0.0104} \\
\hline & 0.0143 & 0.40 & \\
\hline \multirow{8}{*}{6} & 0.0078 & 0.108 & \multirow{8}{*}{0.2073} \\
\hline & 0.0312 & 0.072 & \\
\hline & 0.0143 & 0.252 & \\
\hline & 0.0557 & 0.168 & \\
\hline & 0.2771 & 0.072 & \\
\hline & 0.4591 & 0.168 & \\
\hline & 0.5405 & 0.048 & \\
\hline & 0.6095 & 0.112 & \\
\hline $7-10$ & 0.0078 & 1.00 & 0.6095 \\
\hline
\end{tabular}

Evaluation based on the average belief. According to the paper[6], here we use three indexes to evaluate Plan A and Plan B:

1). Max average belief: $B_{\max }=\max _{t} f_{\text {avg }}(t)$

2). Min time for $B_{\text {max }}: T_{\min }=\min \left\{t \mid \max _{t} f_{\text {avg }}(t)\right\}$

3). Belief of a given moment: $E_{t}=f_{\text {avg }}(t)$

From the belief tables of $\mathrm{G}$, for $B_{\max }$, both of the plans got the same effect. But for $T_{\min }$ and $E_{t}$, Plan A is better than Plan B. and then we an get the conclusion that Plan A is better than Plan B.

\section{Conclusion}

To meet the requirement of target selection and operation evaluation, we introduced the time delay and the random belief sequence in this paper to simulate the uncertain time factors in the military operations, which is called random timed influence nets. The RTIN improved the usability of the timed influence nets. It can also be useful for military to analyze the tasks and make efficient strategy for combating operations.

\section{References}

[1] Rosen J A, Smith WL. Influence net modeling with causal strengths:An evolutionary approach[C]//roceedings of the 1996 Command and Control Research and Technology Symposium, Monterey CA, 1996.

[2] Murphy K. Dynamic Bayesian networks: Representation, inference and learning[D]. UC Berkley, 2002.

[3] Wagenhals L W, Shin I, Levis A H.Creating executable models of influence nets with coloured Petri nets[J].International JournM of STTT, Spring-Verlag,1998, 2(2):168 181.

[4] Haider S, Levis A H. A heuristic approach for belief updating in timed influence nets $[\mathrm{C}] / /$ Proceedings of 2004Command and Control Research and Technology Symposium, San Deigo, 2004.

[5] Haider S, Levis A H. Modeling time-varying uncertain situations using dynamic influence nets[J]. International Journal of Approximate Reasoning, 2008, 49(2):488 502.

[6] Haider S. Levis A H. On finding effective courses of action in a complex situation using evolutionary algorithms[C]/Proceedings of the 10th International Command and Control Research and Technology Symposium, 2005 